\title{
A New Generalized Weighted Weibull Distribution
}

\author{
Salman Abbas \\ Department of Mathematics, \\ COMSATS University Islamabad, Wah Capmus, Pakistan \\ salmanabbas@ciitwah.edu.pk \\ Gamze Ozal \\ Department of Statistics \\ Hacettepe University, Ankara, Turkey \\ gamzeozl@hacettepe.edu.tr \\ Saman Hanif Shahbaz \\ Department of Statistics \\ King Abdulaziz University, Jeddah, Kingdom of Saudi Arabia \\ saman.shahbaz17@gmail.com \\ Muhammad Qaiser Shahbaz \\ Department of Statistics \\ King Abdulaziz University, Jeddah, Kingdom of Saudi Arabia \\ qshahbaz@gmail.com
}

\begin{abstract}
In this article, we present a new generalization of weighted Weibull distribution using Topp Leone family of distributions. We have studied some statistical properties of the proposed distribution including quantile function, moment generating function, probability generating function, raw moments, incomplete moments, probability, weighted moments, Rayeni and $q-t h$ entropy. We have obtained numerical values of the various measures to see the effect of model parameters. Distribution of order statistics for the proposed model has also been obtained. The estimation of the model parameters has been done by using maximum likelihood method. The effectiveness of proposed model is analyzed by means of a real data sets. Finally, some concluding remarks are given.
\end{abstract}

Keywords: Weighted Weibull, Topp Leone family, Order Statistics, Entropy

\section{Introduction}

In some situations, it is found that the classical distributions are not suitable for the data sets related to the field of engineering, financial, biomedical, and environmental sciences. The extension of classical models is, therefore, continually needed to obtain suitable model for applications in these areas. Researchers have obtained several extended models for use in these situations but still the room is available to obtain new models with much wider applicability.

The Weibull distribution is not a suitable model to explain the non-monotone hazard rate function (hrf), such as unimodal, U-shaped or bathtub form. Hence, there are many generalizations of the Weibull distribution in the literature. Some notable models are, exponentiated Weibull distribution by Mudholkar and Srivastava (1993), extended Weibull distribution by Ghitany et al. (2005), beta Weibull distribution by Lee et al. (2007), the flexible Weibull distribution by Bebbington et al. (2007), Kumaraswamy Weibull distribution by Cordeiro et al. (2010), truncated Weibul- 
1 distribution by Zhang and Xie (2011), The Topp-Leone Generated Weibull distribution by Aryal et al. (2016), Generalized weibull distributions by Lai, (2014), The Kumaraswamy-transmuted exponentiated modified Weibull distribution by AlBabtain et al. (2017), Generalized Flexible Weibull Distribution by Ahmad and Iqbal (2017), A reduced new modified Weibull distribution by Almalki (2018), and the transmuted exponentiated additive Weibull distribution by Nofal et al. Nofal et al..

Nasiru (2015) proposed a new Weighted Weibull (WW) distribution and discussed its statistical properties using Azzalani's family of weighted distributions by Azzalini (1985). The density ( $p d f)$ and distribution function $(c d f)$ of the WW distribution are given by

$$
f(x)=\left(1+\lambda^{\gamma}\right) \alpha \gamma x^{\gamma-1} e^{-\alpha x^{\gamma}\left(1+\lambda^{\gamma}\right)} ; \quad x, \alpha, \lambda>0
$$

and

$$
F(x)=1-e^{-\alpha x^{\gamma}\left(1+\lambda^{\gamma}\right)} \quad x, \alpha, \lambda>0
$$

where, $\alpha$ is a scale parameter and $\lambda$ and $\gamma$ are shape parameters. The corresponding survival function of the WW distribution is given by

$$
\bar{F}(x)=e^{-\alpha x^{\gamma}\left(1+\lambda^{\gamma}\right)} .
$$

Note that the WW distribution reduces to Weibull distribution for $\lambda=0$.

Topp-Leone family of distributions is proposed by Al-Shomrani et al. (2016). The cdf of the proposed family is given by

$$
F_{T L-G}(t)=[G(t)]^{b}[2-G(t)]^{b}=\left[1-(\bar{G}(t))^{2}\right]^{b}:, \quad x \epsilon \Re, \quad b>0
$$

and the corresponding pdf is obtained as

$$
f_{T L-R}(t)=2 b g(t) \bar{G}(t)\left[1-(\bar{G}(t))^{2}\right]^{b-1}, \quad b>0 .
$$

where $g(t)=G^{\prime}(t)$ and $\bar{G}(t)=1-G(t)$.

In this paper a new generalization of the WW distribution, the Topp Leone Weighted Weibull (TLWW) distribution, is obtained. The aim of this generalization is to provide a flexible extension of the WW distribution which can be used in much wider situations.

The paper is organized as follows: The $p d f$ and $c d f$ of the proposed model is introduced and several mathematical characteristics are studied in Section 2. Distribution of the order statistics is obtained in Sec. 4. Estimation of the model parameters are done in Sec. 5. The influence of the estimators are evaluated in Sec. 6. The validity of prosed model is on real data is presented in Sec. 7. Some concluding remarks are given in Sec. 8.

\section{Topp-Leone Weighted Weibull Distribution and its Properties}

In this section, we have obtained the $p d f$ and $c d f$ of new model. For this, we consider the survival function of the WW distribution given in (3) and have used it in (4). 
The $c d f$ of the TLWW distribution is obtained as

$$
F(x)=\left[1-\left(e^{-\alpha x^{\gamma}\left(1+\lambda^{\gamma}\right)}\right)^{2}\right]^{b}=\left[1-e^{-2 \alpha x^{\gamma}\left(1+\lambda^{\gamma}\right)}\right]^{b}, \quad b>0 .
$$

The density function is obtained by differentiating (6) and is given as

$$
f(x)=2 b \alpha \gamma\left(1+\lambda^{\gamma}\right) x^{\gamma-1} e^{-2 \alpha x^{\gamma}\left(1+\lambda^{\gamma}\right)}\left[1-e^{-2 \alpha x^{\gamma}\left(1+\lambda^{\gamma}\right)}\right]^{b-1}, \quad x, \alpha, \lambda, \gamma, b>0,
$$

where, $\alpha, \gamma, b$ are shape parameters and $\lambda$ is scale parameter. A random variable $X$ having $p d f(7)$ is denoted as $X \sim T L W W(\alpha, \lambda, \gamma, b)$. The proposed model reduces to Topp Leone Weibull distribution for $\lambda=0$. For $\lambda=0$ and $\gamma=1$, it reduces to Topp Leon Exponential distribution.

The reliability function $(r f)$, which is also known as survival function, is the probability of an item not failing prior to some time $t$. The $r f$ of the TLWW distribution is obtained as $R(x)=1-H(x)$ and is given as

$$
S(x)=1-\left[1-e^{-2 \alpha x^{\gamma}\left(1+\lambda^{\gamma}\right)}\right]^{b} .
$$

The hazard rate function which is also known as, force of mortality in actuarial statistics, Mill's ratio in statistics and intensity function in extreme value theory are important characteristics in reliability theory, It is roughly explained as the conditional probability of failure, given it has survived to the time $t$. The $h r f$ of random variable $X$ is defined as $h(x)=f(x) / R(x)$ and for TLWW distribution it is given as

$$
h(x)=\frac{2 b \alpha \gamma\left(1+\lambda^{\gamma}\right) x^{\gamma-1} e^{-2 \alpha x^{\gamma}\left(1+\lambda^{\gamma}\right)}\left[1-e^{-2 \alpha x^{\gamma}\left(1+\lambda^{\gamma}\right)}\right]^{b-1}}{1-\left[1-e^{-2 \alpha x^{\gamma}\left(1+\lambda^{\gamma}\right)}\right]^{b}} .
$$

The cumulative hrf of the TLWW distribution is given by

$$
H(x)=-\log \left|1-\left[1-e^{-2 \alpha x^{\gamma}\left(1+\lambda^{\gamma}\right)}\right]^{b}\right| .
$$

\subsection{Limiting Behavior}

The behaviors of the $p d f, c d f$ and hrf of TLWW distribution are investigated when $x \rightarrow 0$ and $x \rightarrow \infty$. Therefore, $\lim _{x \rightarrow 0} f(x)$ and $\lim _{x \rightarrow \infty} f(x)$ are given in the following

$$
\begin{aligned}
& \lim _{x \rightarrow 0} f(x)=\lim _{x \rightarrow 0}\left[2 b \alpha \gamma\left(1+\lambda^{\gamma}\right) x^{\gamma-1} e^{-2 \alpha x^{\gamma}\left(1+\lambda^{\gamma}\right)}\left[1-e^{-2 \alpha x^{\gamma}\left(1+\lambda^{\gamma}\right)}\right]^{b-1}\right]=0, \\
& \lim _{x \rightarrow \infty} f(x)=\lim _{x \rightarrow \infty}\left[2 b \alpha \gamma\left(1+\lambda^{\gamma}\right) x^{\gamma-1} e^{-2 \alpha x^{\gamma}\left(1+\lambda^{\gamma}\right)}\left[1-e^{-2 \alpha x^{\gamma}\left(1+\lambda^{\gamma}\right)}\right]^{b-1}\right]=\infty
\end{aligned}
$$

From above it is clear that the proposed model has a unique mode. The limiting behavior of $c d f$ and hrf is given below

$$
\lim _{x \rightarrow 0} f(x)=\lim _{x \rightarrow 0}\left[1-e^{-2 \alpha x^{\gamma}\left(1+\lambda^{\gamma}\right)}\right]^{b}=0
$$


Salman Abbas, Gamze Ozal, Saman Hanif Shahbaz, Muhammad Qaiser Shahbaz

$$
\begin{gathered}
\lim _{x \rightarrow \infty} f(x)=\lim _{x \rightarrow \infty}\left[1-e^{-2 \alpha x^{\gamma}\left(1+\lambda^{\gamma}\right)}\right]^{b}=1, \\
\lim _{x \rightarrow 0} h(x)=\lim _{x \rightarrow 0}\left[\frac{2 b \alpha \gamma\left(1+\lambda^{\gamma}\right) x^{\gamma-1} e^{-2 \alpha x^{\gamma}\left(1+\lambda^{\gamma}\right)}\left[1-e^{-2 \alpha x^{\gamma}\left(1+\lambda^{\gamma}\right)}\right]^{b-1}}{1-\left[1-e^{-2 \alpha x^{\gamma}\left(1+\lambda^{\gamma}\right)}\right]}\right]=0, \\
\lim _{x \rightarrow \infty} h(x)=\lim _{x \rightarrow \infty}\left[\frac{2 b \alpha \gamma\left(1+\lambda^{\gamma}\right) x^{\gamma-1} e^{-2 \alpha x^{\gamma}\left(1+\lambda^{\gamma}\right)}\left[1-e^{-2 \alpha x^{\gamma}\left(1+\lambda^{\gamma}\right)}\right]^{b-1}}{1-\left[1-e^{-2 \alpha x^{\gamma}(1+\lambda \gamma)}\right]}\right]=0 .
\end{gathered}
$$

\subsection{Shape}

The distribution and density functions of the proposed model can be expressed in the form of exponentiated G-distribution. Prudnikov et al. (1986) presented a series representation and is given as

$$
(1+x)^{\alpha}=\sum_{j=0}^{\infty} \frac{(1)^{j} \Gamma(\alpha+1)}{j ! \Gamma(\alpha+1-j)} x^{j}
$$

the distribution function of TLWW distribution is written as follow

$$
F(x)=\sum_{j=0}^{\infty}(-1)^{j} \frac{\Gamma(b+1)}{j ! \Gamma(b+1-j)}\left(e^{\alpha x^{\gamma}\left(1+\lambda^{\gamma}\right)}\right)^{2 j} .
$$

The density of TLWW distribution can also be written in the form of exponentiated distributions and is given as

$$
f(x)=\sum_{j=0}^{\infty} \frac{(-1)^{j} 2 \Gamma(b+1)}{j ! \Gamma(b-j)} \alpha \gamma x^{\gamma-1}\left(1+\lambda^{\gamma}\right)\left(e^{-2 \alpha x^{\gamma}\left(1+\lambda^{\gamma}\right)}\right)^{(j+1)}
$$

We, now, present the plots for the density function of the TLWW distribution for different values of parameters in Figures 1 to 4 . Figure 1 shows that as the value of $b$ increase there is a smooth increase in the peak of the curve and starting point is also shifted. One can clearly see from Figure 2 that $\lambda$ plays opposite role as compared with the $b$. As the values of $b$ increase, the curve shrinks and a rapid decrease occurs in the peak of curve. Figure 3 shows that the distribution is positively skewed for the smaller values of $\gamma$. From Figure 4 it can be seen that $\alpha$ plays important role in shape of the distribution. For smaller values of $\alpha$, the curve is smooth but as the values of $\alpha$ increase great change appears and curve's peak increases abruptly. 

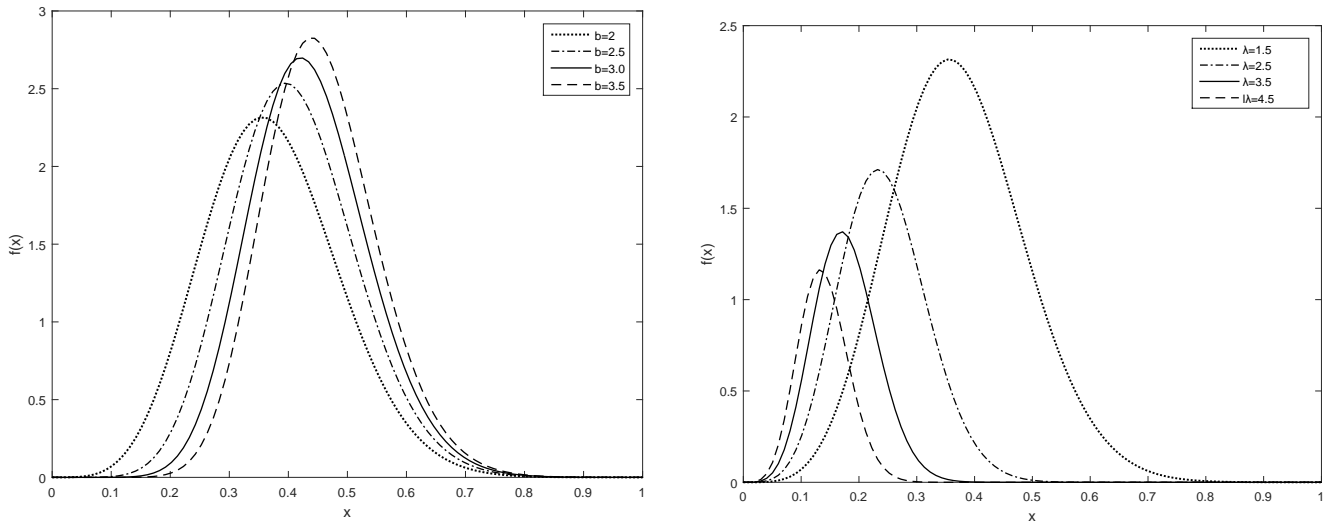

Figure 1: Plots of pdf for $\alpha=2, \gamma=$ Figure 2: Plots of pdf for $b=2, \alpha=$ $2.5, \lambda=1.5$ $2, \gamma=2.5$
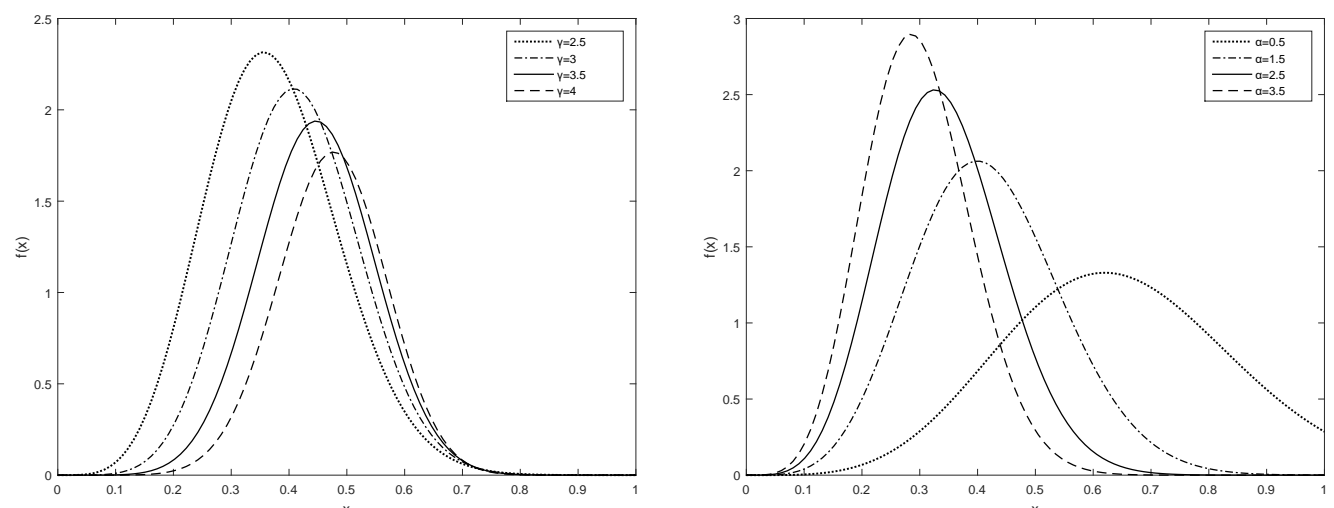

Figure 3: Plots of pdf for $b=2, \alpha=$ Figure 4: Plots of pdf for $b=2, \lambda=$ $2, \lambda=1.5$ $1.5, \gamma=2.5$

\subsection{Quantile Function}

The quantile function of the TLWW distribution is obtained as $Q(u)=F^{-1}(u)$ and given as

$$
Q(u)=-\left(\frac{\ln \left\{1-u^{\frac{1}{b}}\right\}}{2 \alpha\left(1+\lambda^{\gamma}\right)}\right)^{\frac{1}{\gamma}}, \text { for } \alpha>0, u \in(0,1)
$$

Median of the distribution can be obtained by replacing $u=0.5$ in above equation. The quantile function is used to observe the effect of shape parameters on skewness and kurtosis. The Bowley's measure of skewness (S) is given as

$$
S=\frac{Q\left(\frac{1}{4}\right)+Q\left(\frac{3}{4}\right)-2 Q\left(\frac{1}{2}\right)}{Q\left(\frac{3}{4}\right)-Q\left(\frac{1}{4}\right)}
$$

and the Moors's coefficient of kurtosis $(\mathrm{K})$ is given as

$$
K=\frac{Q\left(\frac{7}{8}\right)-Q\left(\frac{5}{8}\right)+Q\left(\frac{3}{8}\right)-Q \frac{1}{8}}{Q\left(\frac{6}{8}\right)-Q\left(\frac{2}{8}\right)} .
$$


Salman Abbas, Gamze Ozal, Saman Hanif Shahbaz, Muhammad Qaiser Shahbaz

\subsection{Moments}

The raw or non-central moment for any probability distribution is obtained by using

$$
\mu_{q}^{\prime}=\int_{-\infty}^{\infty} x^{q} d F(x)
$$

Using $p d f$ of the TLWW distribution in (8), we obtain the raw moments of the TLWW distribution as

$$
\mu_{q}^{\prime}=\int_{0}^{\infty} x^{q} \sum_{j=0}^{\infty} \frac{(-1)^{j} 2 \Gamma(b+1)}{j ! \Gamma(b-j)} \alpha \gamma x^{\gamma-1}\left(1+\lambda^{\gamma}\right)\left(e^{-2 \alpha x^{\gamma}\left(1+\lambda^{\gamma}\right)}\right)^{(j+1)} .
$$

From the transformation $w=(j+1) 2 \alpha x^{\gamma}\left(1+\lambda^{\gamma}\right)$ and after some calculations, the $q-t h$ moment of TLWW distribution is obtained as

$$
\mu_{q}^{\prime}=\sum_{j=0}^{\infty} b_{j} \Gamma\left(1+\frac{q}{\gamma}\right)
$$

where $b_{j}=(-1)^{j} \frac{\Gamma(b+1)}{j ! \Gamma(b-j)}\left(\frac{1}{j+1}\right)^{\frac{q}{\gamma}+1}\left(\frac{1}{2 \alpha\left(1+\lambda^{\gamma}\right)}\right)^{\frac{q}{\gamma}}$. The coefficient of variation $(C V)$, coefficient of skewness $(C S)$, and coefficient of kurtosis $(C K)$ of the TLWW distribution are obtained as follows

$$
\begin{gathered}
C V=\sqrt{\frac{\mu_{2}}{\mu_{1}}-1} \\
C S=\frac{\mu_{3}-3 \mu_{2} \mu_{1}+2 \mu_{1}^{3}}{\left(\mu_{2}-\mu_{1}\right)^{\frac{3}{2}}} \\
C K=\frac{\mu_{4}-4 \mu_{3} \mu_{1}+6 \mu_{2} \mu_{1}^{2}}{\left(\mu_{2}-\mu_{1}^{2}\right)^{2}} .
\end{gathered}
$$

Now, the first incomplete moment is used to derive the mean deviation, Bonferroni, and Lorenz curves. These curves have great influences in economics, reliability, demography, insurance, and medicine. The incomplete moment of the TLWW distribution is obtained by using (7) and is given below.

$$
\varphi_{s}(t)=\int_{0}^{t} x^{s} \sum_{j=0}^{\infty} \frac{(-1)^{j} 2 \Gamma(b+1)}{j ! \Gamma(b-j)} \alpha \gamma x^{\gamma-1}\left(1+\lambda^{\gamma}\right)\left(e^{-2 \alpha x^{\gamma}\left(1+\lambda^{\gamma}\right)}\right)^{(j+1)} d x
$$

Simplifying, the incomplete moments is given by

$$
\varphi_{s}(t)=\sum_{j=0}^{\infty} A_{j}^{*}\left[\gamma\left(1+\frac{s}{\gamma}\right)-\gamma\left(1+\frac{s}{\gamma}\right), 2 \alpha\left(1+\lambda^{\gamma}\right)(j+1)\right] .
$$

where $A_{j}^{*}=(-1)^{j} \frac{\Gamma(b+1)}{j ! \Gamma(b-j)}\left(\frac{1}{j+1}\right)^{\frac{s}{\gamma}+1}\left(\frac{1}{\left(2 \alpha\left(1+\lambda^{\gamma}\right)\right)}\right)^{\frac{s}{\gamma}}$.

The mean deviation about mean $\left[m_{1}=E\left(\left|X-\mu_{1}^{\prime}\right|\right)\right]$ and $\left[m_{2}=E(|X-M|)\right]$ mean deviation about median of $X$ are given as $m_{1}=2 \mu_{1}^{\prime} F\left(\mu_{1}^{\prime}\right)-2 \varphi_{1}\left(\mu_{1}^{\prime}\right)$ and $m_{2}=$ 
$\mu_{1}^{\prime}-2 \varphi_{1}(M)$, respectively, where $\mu_{1}^{\prime}=E(X), M=\operatorname{Median}(X)=Q(0.5)$, and $F\left(\mu_{1}^{\prime}\right)$ is calculated from $(7)$ and $\varphi_{1}(t)$ is the first incomplete moment given by (19) with $s=1$.

These equations for $\varphi_{1}(t)$ can be used to obtain Bonferroni and Lorenz curves given probability $\pi$ as $B(\pi)=\frac{\varphi_{1}(q)}{\pi \mu_{1}^{\prime}}$ and $L(\pi)=\frac{\varphi_{1}(q)}{\mu_{1}^{\prime}}$, respectively, where $\mu_{1}^{\prime}=E(X)$ and $q=Q(\pi)$ is quantile function of $X$ at $\pi$.

The $(q, r)$ th probability weighted moment (PWM) of $X$ is defined as

$$
\rho_{q, r}=\int_{-\infty}^{\infty} x^{q}[F(x)]^{r} f(x) d x .
$$

Using (5) and (6), we can write after some algebra,

$$
[F(x)]^{r} f(x)=\sum_{j, m=0}^{\infty} a(j, m) h_{2 j+1}(x),
$$

where

$$
a(j, m)=\sum_{j, m=0}^{\infty}(-1)^{j+m} \frac{\Gamma(b+1)(r b+1)}{j ! m ! \Gamma(b-j)(r b+1-j)}
$$

and

$$
h_{2 j+1}(x)=2 \alpha \gamma x^{\gamma-1}\left(1+\lambda^{\gamma}\right) e^{-2 \gamma x^{\gamma}\left(1+\lambda^{\gamma}\right)(2 j+1)},
$$

After making transformation, the $(q, r)$ th PWM of $X$ can be expressed as

$$
\rho_{q, r}(x)=a(j, m)\left(\frac{1}{2 j+1}\right)^{\frac{q}{\gamma}+1}\left(\frac{1}{2 \alpha\left(1+\lambda^{\gamma}\right)}\right)^{\frac{q}{\gamma}} \Gamma\left(1+\frac{q}{\gamma}\right) .
$$

Now, we provide numerical values for the mean, variance, coefficient of skewness, and coefficient of kurtosis in Tables 1 to 4, respectively. 
Salman Abbas, Gamze Ozal, Saman Hanif Shahbaz, Muhammad Qaiser Shahbaz

Table 1: Mean of TLWW distribution for $\alpha=1$ and various values of parameters

\begin{tabular}{|c|c|c|c|c|c|c|c|}
\hline \multicolumn{8}{|c|}{$\gamma$} \\
\hline $\mathrm{b}$ & $\bar{\lambda}$ & 4 & 5 & 6 & 7 & 8 & 9 \\
\hline \multirow{6}{*}{1} & 1 & 0.6409 & 0.6958 & 0.7363 & 0.7674 & 0.7919 & 0.8118 \\
\hline & 2 & 0.3754 & 0.3972 & 0.4122 & 0.4232 & 0.4316 & 0.4383 \\
\hline & 3 & 0.2533 & 0.2662 & 0.2754 & 0.2824 & 0.2879 & 0.2923 \\
\hline & 4 & 0.1904 & 0.1998 & 0.2066 & 0.2118 & 0.2159 & 0.2192 \\
\hline & 5 & 0.1524 & 0.1599 & 0.1653 & 0.1694 & 0.1727 & 0.1754 \\
\hline & 6 & 0.1270 & 0.1332 & 0.1378 & 0.1412 & 0.1439 & 0.1461 \\
\hline \multirow{6}{*}{2} & 1 & 0.7429 & 0.7859 & 0.8167 & 0.8397 & 0.8576 & 0.8720 \\
\hline & 2 & 0.4351 & 0.4486 & 0.4572 & 0.4630 & 0.4674 & 0.4708 \\
\hline & 3 & 0.2936 & 0.3007 & 0.3055 & 0.3090 & 0.3117 & 0.3139 \\
\hline & 4 & 0.2206 & 0.2257 & 0.2292 & 0.2318 & 0.2338 & 0.2354 \\
\hline & 5 & 0.1766 & 0.1805 & 0.1833 & 0.1854 & 0.1871 & 0.1884 \\
\hline & 6 & 0.1472 & 0.1505 & 0.1528 & 0.1545 & 0.1559 & 0.1570 \\
\hline \multirow{6}{*}{3} & 1 & 0.7929 & 0.8288 & 0.8541 & 0.8729 & 0.8875 & 0.8990 \\
\hline & 2 & 0.4644 & 0.4731 & 0.4781 & 0.4814 & 0.4837 & 0.4854 \\
\hline & 3 & 0.3134 & 0.3171 & 0.3195 & 0.3212 & 0.3226 & 0.3237 \\
\hline & 4 & 0.2355 & 0.2380 & 0.2397 & 0.2410 & 0.2419 & 0.2427 \\
\hline & 5 & 0.1885 & 0.1904 & 0.1917 & 0.1928 & 0.1936 & 0.1942 \\
\hline & 6 & 0.1571 & 0.1587 & 0.1598 & 0.1606 & 0.1613 & 0.1618 \\
\hline \multirow{6}{*}{4} & 1 & 0.8248 & 0.8557 & 0.8774 & 0.8935 & 0.9058 & 0.9156 \\
\hline & 2 & 0.4830 & 0.4885 & 0.4912 & 0.4927 & 0.4937 & 0.4943 \\
\hline & 3 & 0.3259 & 0.3274 & 0.3282 & 0.3288 & 0.3293 & 0.3296 \\
\hline & 4 & 0.2450 & 0.2457 & 0.2462 & 0.2466 & 0.2469 & 0.2472 \\
\hline & 5 & 0.1961 & 0.1966 & 0.1970 & 0.1973 & 0.1976 & 0.1978 \\
\hline & 6 & 0.1634 & 0.1638 & 0.1641 & 0.1644 & 0.1646 & 0.1648 \\
\hline \multirow{6}{*}{5} & 1 & 0.8477 & 0.8750 & 0.8940 & 0.9080 & 0.9187 & 0.9272 \\
\hline & 2 & 0.4965 & 0.4994 & 0.5004 & 0.5007 & 0.5007 & 0.5006 \\
\hline & 3 & 0.3350 & 0.3347 & 0.3344 & 0.3341 & 0.3339 & 0.3338 \\
\hline & 4 & 0.2518 & 0.2512 & 0.2508 & 0.2506 & 0.2505 & 0.2504 \\
\hline & 5 & 0.2015 & 0.2010 & 0.2007 & 0.2005 & 0.2004 & 0.2003 \\
\hline & 6 & 0.1680 & 0.1675 & 0.1672 & 0.1671 & 0.1670 & 0.1669 \\
\hline \multirow{6}{*}{6} & 1 & 0.8654 & 0.8897 & 0.9066 & 0.9190 & 0.9285 & 0.9360 \\
\hline & 2 & 0.5068 & 0.5078 & 0.5075 & 0.5068 & 0.5060 & 0.5054 \\
\hline & 3 & 0.3420 & 0.3404 & 0.3391 & 0.3382 & 0.3375 & 0.3370 \\
\hline & 4 & 0.2570 & 0.2554 & 0.2544 & 0.2537 & 0.2531 & 0.2527 \\
\hline & 5 & 0.2057 & 0.2044 & 0.2035 & 0.2029 & 0.2025 & 0.2022 \\
\hline & 6 & 0.1715 & 0.1703 & 0.1696 & 0.1691 & 0.1688 & 0.1685 \\
\hline
\end{tabular}


Table 2: Variance of TLWW distribution for $\alpha=1$ and various values of parameters

\begin{tabular}{|c|c|c|c|c|c|c|c|}
\hline \\
\hline b & $\lambda$ & 4 & 5 & 6 & 7 & 8 & 9 \\
\hline \multirow{6}{*}{1} & 1 & 0.0196 & 0.0173 & 0.0151 & 0.0131 & .0114 & .011 \\
\hline & 2 & 0.0249 & 0.0267 & 0278 & 0.0286 & 0291 & 0295 \\
\hline & 3 & 0.0169 & 0.0184 & 0.0194 & 0.0201 & 0.0206 & 0.0211 \\
\hline & 4 & 0.0114 & 0.0124 & 0.0132 & 0.0137 & 0.0142 & 0.0145 \\
\hline & 5 & 0.0081 & 0.0088 & 0.0094 & 0.0098 & 0.0101 & 0.0104 \\
\hline & 6 & 0.0060 & 0.0066 & 0.0070 & 0.0073 & 0.0075 & 0.0077 \\
\hline \multirow{6}{*}{2} & 1 & 0.0144 & 0.0117 & 0.0095 & 0.0079 & 0.0066 & 0.0056 \\
\hline & 2 & 285 & 02 & 0299 & 0.0302 & .0304 & .0306 \\
\hline & 3 & 0.0208 & 0.0216 & 0.0222 & 0.0225 & .0228 & 0.0230 \\
\hline & 4 & 0.0145 & 0150 & 0154 & 57 & .0159 & 0.0161 \\
\hline & 5 & 0.0104 & 0108 & 0.0111 & 0.0113 & 0.0115 & 0.0116 \\
\hline & 6 & 0.0078 & 0.0081 & 0.0083 & 0.0085 & 0.0086 & 0.0087 \\
\hline \multirow{6}{*}{3} & 1 & 0.0109 & 0.0085 & 0.0067 & 0.0054 & 0.0045 & 0.0037 \\
\hline & 2 & 0.0295 & 0.0301 & 0.0305 & 0.0307 & 0.0308 & 0.0309 \\
\hline & 3 & 0.0226 & 0.0231 & 0.0234 & 0.0236 & 0.0237 & 0.0238 \\
\hline & 4 & 0.0159 & 0.0163 & 0.0165 & 0.0166 & 0.0167 & 0.0168 \\
\hline & 5 & 0.0116 & 0.0118 & 0.0119 & 0.0121 & 0.0121 & 0.0122 \\
\hline & 6 & 0.0087 & 0.0089 & 0.0090 & 0.0091 & 0.0091 & 0.0092 \\
\hline \multirow{6}{*}{4} & 1 & 0.0085 & 0.0065 & 0.0050 & 0.0040 & 0.0033 & 0.0027 \\
\hline & 2 & & & & & & 310 \\
\hline & 3 & 0 & 0 & 0.0 & & 0. & 0.0243 \\
\hline & 4 & 0. & 0 & 0.0 & & $0 .($ & 0.0173 \\
\hline & 5 & 0.0 & 4 & 0.0 & 0.1 & 0.0125 & 0.0126 \\
\hline & 6 & 0.0 & 0.0 & 0.0094 & 0.0 & 0.0094 & 0.0095 \\
\hline \multirow{6}{*}{5} & 1 & 0.0 & 1 & 0.0 & 31 & 25 & 0.0021 \\
\hline & 2 & 0.0302 & 0.0306 & 0.0308 & 0.0309 & 0.0310 & 0.0310 \\
\hline & 3 & 0.0244 & 0.0245 & 0.0246 & 0.0246 & 0.0246 & 0.0246 \\
\hline & 4 & 0.0175 & 0.0176 & 0.0176 & 0.0176 & 0.0176 & 0.0176 \\
\hline & 5 & 0.0128 & 0.0128 & 0.0128 & 0.0128 & 0.0128 & 0.0128 \\
\hline & 6 & 0.0097 & 0097 & 0.0097 & .0097 & 0.0097 & 0097 \\
\hline \multirow{6}{*}{6} & 1 & 0.0055 & $\overline{0041}$ & 0.0031 & .0025 & .0020 & 0.0016 \\
\hline & 2 & 0. & 6 & 08 & 0309 & .0310 & 0.0311 \\
\hline & 3 & 0.0249 & 0.0250 & 0.0249 & 0.0249 & 0.0249 & 0.0249 \\
\hline & 4 & 0.0180 & 0.0180 & 0.0179 & 0.0179 & 0.0178 & 0.0178 \\
\hline & 5 & 0.01 & 0.0 & 0.0 & 0.0 & 0.0130 & 0.0130 \\
\hline & 6 & 0.0100 & 0.0099 & 0.0099 & 0.0099 & 0.0098 & 0.0098 \\
\hline
\end{tabular}


Salman Abbas, Gamze Ozal, Saman Hanif Shahbaz, Muhammad Qaiser Shahbaz

Table 3: Coefficient of skewness of the TLWW distribution for $\alpha=1$ and various values of parameters

\begin{tabular}{|c|c|c|c|c|c|c|c|}
\hline \\
\hline b & $\bar{\lambda}$ & 4 & 5 & 6 & 7 & 8 & 9 \\
\hline \multirow{6}{*}{1} & 1 & 2970 & 2545 & 2224 & 1973 & 1772 & 160 \\
\hline & 2 & 1807 & 356 & 47 & 4 & & \\
\hline & 3 & 821 & 754 & 701 & 59 & 624 & 0 \\
\hline & 4 & 0.6295 & 6255 & 6223 & 0.6197 & 6175 & 61 \\
\hline & 5 & 0.6545 & 0.6519 & 0.6498 & 0.6480 & 0.6465 & 0.6452 \\
\hline & 6 & 6692 & 6674 & .6658 & 0.6645 & 0.6634 & 0.6625 \\
\hline \multirow{6}{*}{2} & 1 & 2252 & 1878 & .1609 & 0.1407 & 0.1250 & 0.1124 \\
\hline & 2 & 0.4358 & 4252 & 4184 & .4136 & 0.4100 & 0.4072 \\
\hline & 3 & 0.5555 & .5516 & .5487 & 0.5464 & 0.5446 & 0.5431 \\
\hline & 4 & 0.6123 & 6102 & & & & 0.6050 \\
\hline & 5 & & 3 & 2 & 2 & & 0.6378 \\
\hline & 0 & 66 & 66 & 88 & 31 & 76 & 0.6571 \\
\hline \multirow{6}{*}{3} & 1 & 1904 & 1569 & 4 & 99 & & 919 \\
\hline & 2 & 4119 & 8 & 7 & & & 46 \\
\hline & 3 & 0.5410 & 2 & 9 & 0.5 & 61 & 54 \\
\hline & 4 & 0.6028 & 6021 & 6014 & 0.6 & 0.6 & 9 \\
\hline & 5 & 0.6360 & 6356 & 6352 & 0.6 & 0.6 & 0.6342 \\
\hline & 6 & 0.6556 & 6554 & 6552 & 0.6549 & 0.6547 & 0.6545 \\
\hline \multirow{6}{*}{4} & 1 & 0.1695 & 1386 & 1173 & 0.1016 & 0.0896 & 0.0801 \\
\hline & 2 & 963 & 17 & 94 & 0.3 & & 0.3868 \\
\hline & 2 & 0.5313 & & & & & \\
\hline & 4 & & & & & & \\
\hline & $J$ & 0.6 & & & & & 0.6320 \\
\hline & 0 & 23 & 27 & 28 & 0.6 & 28 & 0.6528 \\
\hline \multirow{6}{*}{5} & 1 & & & & & & \\
\hline & 2 & & & & & & \\
\hline & 3 & 05 & 0 & 0. & 0.5 & 69 & 0. \\
\hline & 4 & & 0.5928 & 0. & 0.5 & 0.5 & 0.5945 \\
\hline & 5 & 0.6281 & 1 & 0. & 0.6 & 0.6 & 0.6304 \\
\hline & 6 & 0.6498 & 6506 & 0.6511 & 0.6513 & 0.6515 & 0.6516 \\
\hline \multirow{6}{*}{6} & 1 & 0.1460 & 1181 & 0.0992 & 0.0855 & 0.0752 & 0.0671 \\
\hline & 2 & 61 & 50 & 3753 & 0.3759 & 0.3765 & 0.3771 \\
\hline & 3 & 5186 & 5207 & 5222 & 0.5232 & 0.5239 & 0.5245 \\
\hline & 2 & & & & & & \\
\hline & 5 & C. & 0 & 8 & 0.6 & 0.6 & 0.6291 \\
\hline & 0 & 0.6479 & 0.6490 & 0.6497 & 0.6502 & 0.6505 & 0.6507 \\
\hline
\end{tabular}


Table 4: Coefficient of kurtosis of TLWW distribution for $\alpha=1$ and various values of parameters

\begin{tabular}{|c|c|c|c|c|c|c|c|}
\hline & & & & $\gamma$ & & & \\
\hline $\mathrm{b}$ & $\lambda$ & 4 & 5 & 6 & 7 & 8 & 9 \\
\hline \multirow{6}{*}{1} & 1 & 1.8001 & 1.7602 & 1.7333 & 1.7144 & 1.7006 & 1.6903 \\
\hline & 2 & 2.0269 & 2.0074 & 1.9933 & 1.9827 & 1.9743 & 1.9675 \\
\hline & 3 & 2.1708 & 2.1619 & 2.1549 & 2.1492 & 2.1445 & 2.1406 \\
\hline & 4 & 2.2366 & 2.2316 & 2.2275 & 2.2240 & 2.2212 & 2.2187 \\
\hline & 5 & 2.2700 & 2.2668 & 2.2641 & 2.2619 & 2.2599 & 2.2583 \\
\hline & 6 & 2.2889 & 2.2867 & 2.2848 & 2.2832 & 2.2818 & 2.2807 \\
\hline \multirow{6}{*}{2} & 1 & 1.7357 & 1.7077 & 1.6903 & 1.6788 & 1.6708 & 1.6651 \\
\hline & 2 & 1.9677 & 1.9547 & 1.9463 & 1.9404 & 1.9360 & 1.9325 \\
\hline & 3 & 2.1343 & 2.1292 & 2.1254 & 2.1224 & 2.1200 & 2.1180 \\
\hline & 4 & 2.2137 & 2.2111 & 2.2089 & 2.2071 & 2.2056 & 2.2044 \\
\hline & 5 & 2.2547 & 2.2531 & 2.2517 & 2.2505 & 2.2495 & 2.2487 \\
\hline & 6 & 2.2780 & 2.2769 & 2.2759 & 2.2751 & 2.2744 & 2.2738 \\
\hline \multirow{6}{*}{3} & 1 & 1.7095 & 1.6879 & 1.6749 & 1.6666 & 1.6609 & 1.6568 \\
\hline & 2 & 1.9372 & 1.9287 & 1.9238 & 1.9206 & 1.9183 & 1.9166 \\
\hline & 3 & 2.1142 & 2.1120 & 2.1105 & 2.1092 & 2.1081 & 2.1072 \\
\hline & 4 & 2.2008 & 2.2001 & 2.1993 & 2.1986 & 2.1980 & 2.1974 \\
\hline & 5 & 2.2460 & 2.2456 & 2.2452 & 2.2447 & 2.2443 & 2.2440 \\
\hline & 6 & 2.2718 & 2.2716 & 2.2713 & 2.2710 & 2.2707 & 2.2705 \\
\hline \multirow{6}{*}{4} & 1 & 1.6955 & 1.6776 & 1.6671 & 1.6604 & 1.6560 & 1.6528 \\
\hline & 2 & 1.9176 & 1.9124 & 1.9098 & 1.9084 & 1.9075 & 1.9068 \\
\hline & 3 & 2.1008 & 2.1008 & 2.1009 & 2.1008 & 2.1007 & 2.1005 \\
\hline & 4 & 2.1921 & 2.1928 & 2.1931 & 2.1931 & 2.1931 & 2.1930 \\
\hline & 5 & 2.2400 & 2.2407 & 2.2409 & 2.2410 & 2.2410 & 2.2410 \\
\hline & 6 & 2.2676 & 2.2681 & 2.2683 & 2.2683 & 2.2683 & 2.2683 \\
\hline \multirow{6}{*}{5} & 1 & 1.6870 & 1.6714 & 1.6624 & 1.6568 & 1.6531 & 1.6505 \\
\hline & 2 & 1.9036 & 1.9008 & 1.9000 & 1.8998 & 1.8999 & 1.9001 \\
\hline & 3 & 2.0909 & 2.0927 & 2.0939 & 2.0948 & 2.0953 & 2.0957 \\
\hline & 4 & 2.1856 & 2.1875 & 2.1885 & 2.1892 & 2.1896 & 2.1899 \\
\hline & 5 & 2.2356 & 2.2370 & 2.2378 & 2.2383 & 2.2386 & 2.2388 \\
\hline & 6 & 2.2644 & 2.2655 & 2.2660 & 2.2664 & 2.2666 & 2.2668 \\
\hline \multirow{6}{*}{6} & 1 & 1.6814 & 1.6673 & 1.6594 & 1.6544 & 1.6512 & 1.6489 \\
\hline & 2 & 1.8928 & 1.8919 & 1.8925 & 1.8934 & 1.8942 & 1.8950 \\
\hline & 3 & 2.0831 & 2.0863 & 2.0886 & 2.0901 & 2.0912 & 2.0921 \\
\hline & 4 & 2.1805 & 2.1833 & 2.1850 & 2.1861 & 2.1869 & 2.1875 \\
\hline & 5 & 2.2320 & 2.2341 & 2.2354 & 2.2362 & 2.2368 & 2.2372 \\
\hline & 6 & 2.2618 & 2.2634 & 2.2643 & 2.2649 & 2.2653 & 2.2656 \\
\hline
\end{tabular}


Salman Abbas, Gamze Ozal, Saman Hanif Shahbaz, Muhammad Qaiser Shahbaz

\subsection{Moment and Probability Generating Function}

Let $X \sim T L W W(b, \alpha, \lambda, \gamma)$, then the moment generating function of $X$ is denoted as $M_{X}(t)$ and is given as

$$
M_{X}(t)=\int_{0}^{\infty} e^{t x} f(x) d x
$$

Using Taylor series and from (7), we have

$$
M_{X}(t)=\sum_{i, j=0}^{\infty} \frac{t^{i}}{i} \frac{(-1)^{j} \Gamma(b+1)}{j ! \Gamma(b-j)}\left(\frac{1}{j+1}\right)^{\frac{i}{\gamma}+1}\left(\frac{1}{\left(2 \alpha\left(1+\lambda^{\gamma}\right)\right.}\right)^{\frac{i}{\gamma}} \Gamma\left(1+\frac{i}{\gamma}\right) .
$$

Similarly, if $X \sim T L W W(b, \alpha, \lambda, \gamma)$, then the probability generating function of $X$ is denoted as $P h i_{X}(t)$ and is given as

$$
\Phi_{X}(t)=\int_{0}^{\infty} t^{x} f(x) d x
$$

Using

$$
M_{X}(t)=\sum_{l=0}^{\infty} \frac{(\ln t)^{l} x^{l}}{l !}
$$

we have,

$$
\Phi_{X}(t)=\sum_{l, j=0}^{\infty} \frac{(\ln t)^{l}}{l !} \frac{)^{j} \Gamma(b+1)}{j ! \Gamma(b-j)}\left(\frac{1}{j+1}\right)^{\frac{i}{\gamma}+1}\left(\frac{1}{2 \alpha\left(1+\lambda^{\gamma}\right)}\right)^{\frac{l}{\gamma}} \Gamma\left(1+\frac{l}{\gamma}\right)^{\frac{l}{\gamma}}
$$

\subsection{Order Statistics}

The order statistics play an important role in the statistical theory. Let $X_{1}, X_{2},,,,, X_{n}$ be a random samples from the TLWW distribution, then the density function of the $i-$ th order statistics is given as

$$
f_{i: n}(x)=\sum_{j=0}^{\infty} \psi_{j}\left(e^{-2 \alpha x^{\gamma}\left(1+\lambda^{\gamma}\right)}\right)^{m+1},
$$

where

$$
\psi_{j}=\frac{n !}{(i-1) !(n-i) !} 2 \alpha \gamma\left(1+\lambda^{\gamma}\right) x^{\gamma-1} \sum_{m=0}^{b(i+j)-1}\left(\begin{array}{c}
n-1 \\
j
\end{array}\right)\left(\begin{array}{c}
b(i+j)-1 \\
m
\end{array}\right) .
$$


The pdf of the first order statistics can be obtained by using $i=1$ and the pdf of $n t h$ order statistics can be defined by replacing $i=n$.

\section{Entropy}

The entropy is used to explain the existence of uncertainty in the random variables. Different method to measure the uncertainty in random variables are developed by researchers. Reyni's entropy is obtained by using

$$
I_{p}=\frac{1}{1-p} \log \int_{-\infty}^{\infty} f(x)^{p} d x, \quad p>0, p \neq 0
$$

Now using Eq. (8), we have

$$
I_{p}=\frac{1}{1-p} \log \int_{-\infty}^{\infty}\left\{\sum_{j=0}^{\infty} \frac{(-1)^{j} 2 \Gamma(b+1)}{j ! \Gamma(b-j)} \alpha \gamma x^{\gamma-1}\left(1+\lambda^{\gamma}\right)\left(e^{-2 \alpha x^{\gamma}\left(1+\lambda^{\gamma}\right)}\right)^{(j+1)}\right\}^{p} d x
$$

To obtained the expression of entropy, we assume $z=2 \alpha x^{\gamma}\left(1+\lambda^{\gamma}\right)(j+1)$, and after simplification we have

$$
I_{p}=\frac{1}{1-p} \log \left\{\kappa_{j}^{p} A^{*} \Gamma(p+1)\right\}
$$

where

$$
\begin{aligned}
& \kappa=\sum_{j=0}^{\infty} \frac{(-1)^{j} 2 \Gamma(b+1)}{j ! \Gamma(b-j)} \alpha \gamma x^{\gamma-1}\left(1+\lambda^{\gamma}\right) \\
& A^{*}=\alpha^{p}\left(\frac{1}{2 \alpha\left(1+\lambda^{\gamma}\right)(j+1)}\right)^{p}
\end{aligned}
$$

Next, the $q-$ th entropy is define as

$$
H_{q}=\frac{1}{q-1} \log \left\{1-\int_{-\infty}^{\infty} f(x)^{q} d x\right\}
$$

So, the $q-$ th entropy for the TLWW distribution is given as

$$
H_{q}=\frac{1}{q-1} \log \left\{1-\kappa_{j}^{q} A^{*} \Gamma(q+1)\right\}
$$

where

$$
\begin{aligned}
& \kappa=\sum_{j=0}^{\infty} \frac{(-1)^{j} 2 \Gamma(b+1)}{j ! \Gamma(b-j)} \alpha \gamma x^{\gamma-1}\left(1+\lambda^{\gamma}\right) \\
& A^{*}=\alpha^{p}\left(\frac{1}{2 \alpha\left(1+\lambda^{\gamma}\right)(j+1)}\right)^{q}
\end{aligned}
$$




\section{Estimation}

In this section, we have discussed estimation of the parameters of TLWW distribution. Let $x_{1}, x_{2},,,, x_{n}$ be the random sample of size $n$ from TLWW distribution. The log likelihood function is, then, given as

$$
\begin{aligned}
L= & n \log (2)+n \log (b)+n \log (\alpha)+n \log (\gamma)+n \log \left(1+\lambda^{\gamma}\right)+(\gamma-1) \sum_{i=0}^{n} x_{i} \\
& -2 \alpha\left(1+\lambda^{\gamma}\right) \sum_{i=0}^{n} x_{i}^{\gamma}+(b-1) \sum_{i=0}^{n} \log \left(1-e^{-2 \alpha x^{\gamma}\left(1+\lambda^{\gamma}\right)}\right) .
\end{aligned}
$$

The parameters are obtained by solving the nonlinear equations which are obtained by differentiating (11). The score vector components, say $U(\theta)=\left(\frac{d l}{d b}, \frac{d l}{d \alpha}, \frac{d l}{d \gamma}, \frac{d l}{d \lambda}\right)^{T}=$ $\left(U_{b}, U_{\alpha}, U_{\gamma}, U_{\lambda}\right)^{T}$, are given as follows:

$$
\begin{gathered}
U_{\alpha}=\frac{n}{\alpha}-2\left(1+\lambda^{\gamma}\right) \sum_{i=0}^{n} x_{i}+(b-1) \sum_{i=0}^{n} \frac{2 x_{i}^{\gamma}\left(1+\lambda^{\gamma}\right) w_{i}}{1-w_{i}}, \\
U_{b}=\frac{n}{b}+\sum_{i=0}^{n} \log \left(1-w_{i}\right), \\
U_{\gamma}=\frac{n}{\gamma}+\frac{n \lambda^{\gamma} \log (\lambda)}{1+\lambda^{\gamma}}-2 \alpha \lambda^{\gamma} \log (\lambda) \sum_{i=0}^{n} x_{i}^{\gamma}+\sum_{i=0}^{n} \log \left(x_{i}\right)-2 \alpha(1+\lambda) \sum_{i=0}^{n} x_{i}^{\gamma} \log \left(x_{i}\right) \\
-(b-1) \sum_{i=0}^{n} \frac{w_{i}\left(2 x^{\gamma} \alpha(1+\lambda) \log \left(x_{i}\right)-2 x^{\gamma} \alpha \lambda^{\gamma} \log (\lambda)\right.}{1-w_{i}}, \\
U_{\lambda}=\frac{n \gamma \lambda^{\gamma-1}}{1+\lambda^{\gamma}} 2 \alpha \gamma \lambda^{\gamma-1} \sum_{i=0}^{n} x_{i}^{\gamma}+(b-1) \sum_{i=0}^{n} \frac{2 x^{\gamma} \alpha \gamma \lambda^{\gamma-1} w_{i}}{1-w_{i}},
\end{gathered}
$$

where $w_{i}=e^{-2 \alpha x^{\gamma}\left(1+\lambda^{\gamma}\right)}$. The maximum likelihood estimate for the parameters are obtained by equating the above expressions equal to zero. For interval estimation of the parameters, we obtain the $4 \times 4$ observed information matrix given by

$$
J(\theta)=-\left(\begin{array}{cccc}
J_{b b} & J_{b \alpha} & J_{b \gamma} & J_{b \lambda} \\
& J_{\alpha \alpha} & J_{\alpha \gamma} & J_{\alpha \lambda} \\
& & J_{\gamma \gamma} & J_{\gamma \lambda} \\
& & & J_{\lambda \lambda}
\end{array}\right)
$$

The entries of $J(\theta)$ can be easily computed numerically. Under standard regularity conditions when $n \longrightarrow \infty$, the distribution of $\hat{\theta}$ can be approximated by a multivariate normal $N_{4}=\left(0, J(\hat{\theta})^{-1}\right)$ distribution to construct approximate confidence intervals for the parameters. Here, $J(\hat{\theta})$ is the observed information matrix evaluated at $\theta$. 


\section{Application}

In this section, we show the performance of the proposed model with a real data set. We use the distributions in Table 5 to compare the existing distributions with the proposed distribution. The comparison has been done by fitting various models on

Table 5: Fitted Distributions and their Abbreviations

\begin{tabular}{ccc}
\hline Distributions & Abb & Referrence \\
\hline Topp Leone Weighted Weibull & TLWW & Proposed \\
Kumaraswamy Weibull & KwW & $(10)$ \\
Exponential Weibull & ExW & $(9)$ \\
Exponentiated Weibull-Exponential & ExpWEx & $(11)$ \\
Marshall-Olkin exponential-Weibull & MOEW & $(18)$ \\
Weighted Weibull & WW & $(16)$ \\
\hline
\end{tabular}

real data sets and comparing the goodness of fit measures. In order to compare the fit of TLWW model with other models, we provide the values of the Akaike Information Criterion (AIC) and Consistent Akaike Information Criterion (CAIC).

The data have been obtained from Bjerkedal by Bjerkedal et al. (1960) and represents the survival times (in days) of 72 guinea pigs infected with virulent tubercle bacilli. The data set is given in Table 6 .

Table 6: Data for the survival time (9n days) of 72 guinea pigs infected with virulent tubercle bacilli

\begin{tabular}{cccccccccccc}
\hline 0.1 & 0.33 & 0.44 & 0.56 & 0.59 & 0.72 & 0.74 & 0.77 & 0.92 & 0.93 & 0.96 & 1 \\
1 & 1.02 & 1.05 & 1.07 & 07 & .08 & 1.08 & 1.08 & 1.09 & 1.12 & 1.13 & 1.15 \\
1.16 & 1.2 & 1.21 & 1.22 & 1.22 & 1.24 & 1.3 & 1.34 & 1.36 & 1.39 & 1.44 & 1.46 \\
1.53 & 1.59 & 1.6 & 1.63 & 1.63 & 1.68 & 1.71 & 1.72 & 1.76 & 1.83 & 1.95 & 1.96 \\
1.97 & 2.02 & 2.13 & 2.15 & 2.16 & 2.22 & 2.3 & 2.31 & 2.4 & 2.45 & 2.51 & 2.53 \\
2.54 & 2.54 & 2.78 & 2.93 & 3.27 & 3.42 & 3.47 & 3.61 & 4.02 & 4.32 & 4.58 & 5.55 \\
\hline
\end{tabular}

In Table 7 , the values of estimated parameters and the statistic are given. We can easily see that the proposed model provide smaller values of the computed statistics which indicate that it provides better fit than competing models.

Table 7: The MLEs and the values of AIC, CAIC and -2LL statistics

\begin{tabular}{cccccccc}
\hline Model & \multicolumn{3}{c}{ Parameters } & & $-2 L L$ & $A I C$ & CI AC \\
\hline \hline TLWW & $\alpha$ & $\lambda$ & $\gamma$ & $\lambda$ & & & \\
KwW & 1.655 & 2.037 & 1.157 & 3.055 & 205.62 & 213.62 & 214.217 \\
ExW & 1.617 & 0.311 & $1.2 \times 10^{-8}$ & - & 208.034 & 214.034 & 214.387 \\
ExpWEx & 0.409 & 1.958 & 2.082 & 0.127 & 304.341 & 312.341 & 312.938 \\
MOEW & 0.091 & 0.012 & 2.940 & - & 245.993 & 251.993 & 252.346 \\
WW & 0.213 & 0.621 & 1.617 & - & 208.034 & 214.034 & 214.387 \\
\hline
\end{tabular}

Figures 5 and 6 shows the plots for the fitted models and probability plot of the TLWW distribution, respectively.

As seen from Figures 5 and 6, the proposed model adequately fits the data as compared with other models. 
Salman Abbas, Gamze Ozal, Saman Hanif Shahbaz, Muhammad Qaiser Shahbaz
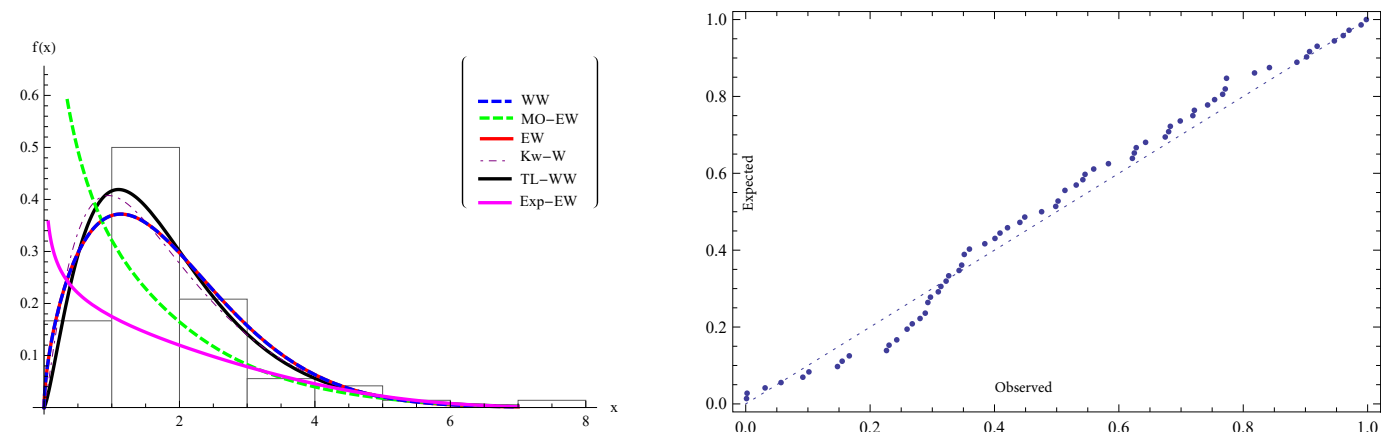

Figure 5: Plots for the fitted models Figure 6: Probability Plot of TLWW for the data set

distribution for the data set

\section{Conclusion}

This paper presents the Topp Leone weighted Weibull distribution. We have obtained various properties for the proposed model; including, shape, moments, moment generating function, probability generating moments, and reliability analysis. The effects of model parameters are discussed in terms of mean, variance, coefficient of skewness, and coefficient of kurtosis. The method of maximum likelihood is used for the estimation of the parameters. We study the data regarding the survival time of 72 Pigs (in days) for the evaluation of the proposed model in practical application. The results show that the proposed model is a better fit as compared with the available models for modeling of the used data.

\section{References}

1. Ahmad, Z. and Iqbal, B. (2017). Generalized flexible weibull extension distribution. Circulation in Computer, 2(4):68-75.

2. Al-Babtain, A., Fattah, A. A., Ahmed, A.-H. N., and Merovci, F. (2017). The kumaraswamy-transmuted exponentiated modified weibul1 distribution. Communications in Statistics-Simulation and Computation, 46(5):3812-3832.

3. Al-Shomrani, A., Arif, O., Shawky, A., Hanif, S., and Shahbaz, M. Q. (2016). Topp-leone family of distributions: some properties and application. Pakistan Journal of Statistics and Operation Research, 12(3):443-451.

4. Almalki, S. J. (2018). A reduced new modified weibull distribution. Communications in Statistics-Theory and Methods, 47(10):2297-2313.

5. Aryal, G. R., Ortega, E. M., Hamedani, G., and Yousof, H. M. (2016). The topp-leone generated weibull distribution: regression model, characterizations and applications. International Journal of Statistics and Probability, $6(1): 126$.

6. Azzalini, A. (1985). A class of distributions which includes the normal ones. Scandinavian journal of statistics, pages 171-178. 
7. Bebbington, M., Lai, C.-D., and Zitikis, R. (2007). A flexible weibull extension. Reliability Engineering \& System Safety, 92(6):719-726.

8. Bjerkedal, T. et al. (1960). Acquisition of resistance in guinea pies infected with different doses of virulent tubercle bacilli. American Journal of Hygiene, 72(1):130-48.

9. Cordeiro, G. M., Ortega, E. M., and Lemonte, A. J. (2014). The exponential-weibull lifetime distribution. Journal of Statistical Computation and Simulation, 84(12):2592-2606.

10. Cordeiro, G. M., Ortega, E. M., and Nadarajah, S. (2010). The kumaraswamy weibull distribution with application to failure data. Journal of the Franklin Institute, 347(8):1399-1429.

11. Elgarhy, M., S. M. and Kibria, B. (2017). Exponentiated weibullexponential distribution with applications. APPLICATIONS AND APPLIED MATHEMATICS-AN INTERNATIONAL JOURNAL, 12(2):710725.

12. Ghitany, M., Al-Hussaini, E., and Al-Jarallah, R. (2005). Marshall-olkin extended weibull distribution and its application to censored data. Journal of Applied Statistics, 32(10):1025-1034.

13. Lai, C.-D. (2014). Generalized weibull distributions. In Generalized Weibull Distributions, pages 23-75. Springer.

14. Lee, C., Famoye, F., and Olumolade, O. (2007). Beta-weibull distribution: some properties and applications to censored data. Journal of modern applied statistical methods, 6(1):17.

15. Mudholkar, G. S. and Srivastava, D. K. (1993). Exponentiated weibull family for analyzing bathtub failure-rate data. IEEE transactions on reliability, 42(2):299-302.

16. Nasiru, S. (2015). Another weighted weibull distribution from azzalinis family. European Scientific Journal, ESJ, 11(9).

17. Nofal, Z. M., Afify, A. Z., Yousof, H. M., Granzotto, D. C. T., and Louzada, F. (2018). The transmuted exponentiated additive weibull distribution: properties and applications. Journal of Modern Applied Statistical Methods, $17(1): 4$.

18. Pogány, T. K., Saboor, A., and Provost, S. (2015). The marshall-olkin exponential weibull distribution. Hacettepe Journal of Mathematics and Statistics, 44(6):1579.

19. Zhang, T. and Xie, M. (2011). On the upper truncated weibull distribution and its reliability implications. Reliability Engineering 85 System Safety, 96(1):194-200. 